IJICIS, Vol.21, No.3, 1-12

DOI: 10.21608/ijicis.2021.77345.1094

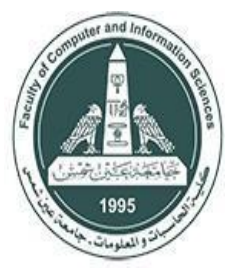

International Journal of Intelligent Computing and Information Sciences

https://ijicis.journals.ekb.eg/

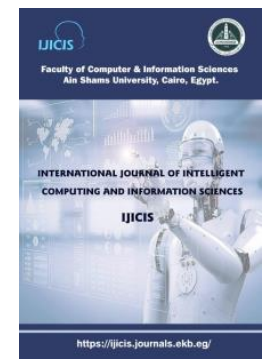

\title{
ONTOLOGY-BASED APPROACH FOR FEATURE LEVEL SENTIMENT ANALYSIS
}

\author{
Eman M. Aboelela* \\ Information Systems Department, \\ Faculty of Computer and \\ Information Sciences, Ain Shams \\ University, \\ Cairo, Egypt. \\ eman_aboelela@cis.asu.edu.eg
}

\author{
Walaa Gad
}

Information Systems Department,

Faculty of Computer and

Information Sciences, Ain Shams

University,

Cairo, Egypt.

walaagad@cis.asu.edu.eg

\section{Rasha Ismail}

Received 2021- 5-24; Revised 2021-8-11; Accepted 2021-8-11

Abstract: Through the state-of-the-art digitalization, we can see a massive growth in user-generated content on the web that provides feedback from people on a variety of topics. However, manually managing large-scale user feedback would be a difficult task and a waste of time. Therefore, the concept of sentiment analysis is emerged. Sentiment analysis is a computerized study of individuals' feelings and opinions about an entity or product. It can be executed at three different levels: document level, sentence or phrase level, and feature level. This paper proposes a novel ontologybased approach for feature level sentiment analysis. The proposed approach extracts the product features using semantic similarity and Wordnet ontology and uses the SentiWordent dictionary to classify the users' comments as positive and negative. Furthermore, it manages negative words to obtain more precise classification results. The proposed approach is assessed by using two benchmark amazon products' datasets in terms of accuracy; recall, precision, and f-measure. The performance reaches to $92.4 \%$ accuracy, $97.2 \%$ precision, $92.8 \%$ recall, and $94.4 \%$-measure.

Keywords: Sentiment Analysis, Wordnet Ontology, SentiWordnet, and Semantic Similarity.

\footnotetext{
* Corresponding author: Eman M. Aboelela Information Systems Department, Faculty of Computer and Information Sciences, Ain Shams University, Cairo, Egypt E-mail address: eman_aboelela@cis.asu.edu.eg
} 


\section{Introduction}

Today, sentiment analysis has become a very useful tool, especially during the COVID-19 pandemic. By analyzing student feedback/opinions/ reviews to help schools, e-learning platforms and teachers know what features they can enhance to improve the performance of the e-learning process [1]. Sentiment analysis is made up of three main components: feedback holder, feedback target, feedback polarity [2]. The feedback holder is the opinion owner and may be an individual or organization. The feedback target is an entity-related opinion that may be a topic, product, service or event. Feedback polarity is a class label for positive or negative opinion. There are two types of webbased reviews that are structured and unstructured [3]. Structured reviews are written in the form of pros and cons to determine what is positive and negative in the service or product. Unstructured reviews are written in a human natural language so they may contain untruthful words. The sentiment analysis analyses the reviews according to three levels of analysis, namely document/review level, sentence/phrase level and aspect/feature level [4]. The document level sentiment analysis considers the entire review as a source of opinions on a single entity. It classifies the whole review as a positive or negative label. Sentiment analysis at the sentence level considers each sentence as a separate source that includes a single opinion. It classifies each sentence as either positive or negative. Feature level sentiment analysis is the most valuable level of analysis in decision-making. Since it classifies the entire review in a positive or negative label based on each feature. This is useful for identifying the positive and negative features of the product or service. Two types of product features are discussed in the feature level sentiment analysis, which are implicit and explicit [3]. Implicit features are derived from the meaning of the sentence, but explicit features are explicitly referred to in the sentence. Two major tasks are involved in sentiment analysis at the feature level, which are the extraction of explicit features from unstructured reviews and the classification of reviews. There are several challenges facing sentiment analysis and causing poor classification performance [5]. Some of these challenges are multiple languages of user comments, fake comments, manipulation of emoticons, implicit features, spam features, and negative words that change the class label of opinion words. Therefore, in this paper a novel ontology-based approach for feature level sentiment analysis is proposed to address some of these challenges. While, the proposed approach uses semantic similarity and Wordnet Ontology to find the domain product features and avoid spamming. It considers only the comments that contain a product feature to avoid the fake comments. In addition, the proposed approach considers the domain product features' synonyms, superior, and inferior words as product features. Additionally, negations are considered in the proposed approach to accurately classify comments. To obtain more precise polarity scores, the proposed approach uses a corpus of positive and negative sentiments with the SentiWordnet dictionary. The experiments show promising performance in terms of average accuracy, recall, precision, and f-measure. The rest of the paper is organized as follow. Section 2 presents related work. The proposed approach is introduced and explained in section 3. In section 4, experiments and results are shown. Conclusion and future work are discussed in section 5.

\section{Related Work}

In [6], Nouns and noun phrases are considered as product features, and adjectives are opinion words. Syntactic parser and Stanford dependency parser are used to detect Part Of Speech information of each word and obtain the syntactic relationships between the words in the sentence. In addition, 
SentiWordnet is used to detect the opinion word polarity. If the opinion word does not exist in SentiWordnet, then its synonyms are found with the help of Wordnet.

In [7], three stages are introduced: firstly, construct a lexicon of text components with semantic orientation. Secondly, apply a lexicon-based sentiment analysis to classify customer reviews. Lastly, assess classification outcomes. Customer feedback is gathered from TripAdvisor.

In [8], different methods of classification of feelings about the documents were evaluated. The researchers used the Naive Bayes, Support Vector Machine, Pointwise Mutual Information (PMI) and SentiWordnet dictionary to analyze the opinions expressed for film reviews.

The authors in [9] introduced a recommendation technique based on sentiment analysis to recommend the top 10 books on various computer science disciplines. The authors were interested in books on the topics of "cloud computing". The technique started with a study of cloud-based books. The features of the books are classified according to the analysis of customers' needs and their collected reviews. Categorized features are weighted according to their significance and use. Finally, the top ten books on cloud computing topics are ranked.

In [10], a general category of discriminative models depending on recurrent neural networks (RNNs) and word embedding is proposed. Numerous RNN architectures are used like Elman-RNN, Jordan-RNN, short-term memory (LSTM). Other linguistic features are included in RNN architecture. The authors used word embedding of Google, SENNA, and Amazon. Experiments showed that RNNs outperformed conditional random fields (CRFs) and LSTM RNN achieved the best results.

In [11], a deep learning-based approach is proposed. The authors used a seven layer deep convolutional neural network to tag features and non-features words. The seven layers are: the input layer, two convolution layers, each with a max-pooling layer; a fully connected layer, and the output layer. In addition, a set of heuristic linguistic patterns is used and integrated with the neural network for the feature extraction. The approach performed better than the state-of-the-art methods.

The authors in [12] improved the method presented in [9] for extracting and analyzing features. Whereas, the method in [9] used the features categorization technique in a book recommendation without applying any features validation. Therefore, the features in are analyzed and modified to be frequency of occurrence in search engine results page (SERP), useful content, extraneous content, enough material, physical attributes, market availability, and price. The categorized book features and human intelligence are used for online reviews to help the users in finding the books they need. Precision is used to assess the presented method.

A domain sentiment lexicon was developed by Yang et al. in [13]. The resulting dictionary and external textual data were used for the sentiment analysis. They introduced a hybrid approach and compared their approach with simple baseline models.

A feature-based opinion mining technique is proposed in [14] to recommend books for university syllabus. The authors used the features presented in [12] and developed the technique presented in [9]. User feedback is collected from highly rated online merchandiser books worldwide and from various websites instead of analyzing the comments from specific thematic books. Features are retrieved from reviews based on their adjectives and indicative terms used by users. The authors presented an algorithm for the classification of sentiment features according to human intelligence and examined the reciprocal meaning of opinion words. Then, the orientation of each feature is identified. In addition, the weights are assigned to the features based on the importance suggested by the users in their opinions. Finally, the books are ranked and the recommendation made.

A novel Convolutional Neural Network (CNN) model with two types of embeddings is introduced in [15]. Embeddings used are both general purpose embedding and domain-specific 
embedding. The authors used a pure CNN model to label the sequences. The model used two embedding layers, four CNN layers, a fully connected shared layer, and a softmax layer. The model presented was successful and outperformed the most modern methods.

A recommendation model based on a weighted feature-based sentiment analysis using a deep learning approach is proposed in [16]. The model had two main components: the feature-based sentiment analysis component and the recommendation generation component. The first component used a multi-channel convolutional neural network model to extract the product features and generate feature rating matrix. The multi-channel convolutional neural network model consists of two entry layers called word embedding and the POS tag embedding. The second used a tensor factorization (TF) approach to calculate the weighted feature ratings and infer the overall rating prediction. The model achieved better results than the baselines.

Some of the previous studies are lexicon-based studies, but they only used the sentiment lexicon which is not enough to get more precise polarity scores. Unlike the proposed ontology-based approach for feature level sentiment analysis that uses a sentiment lexicon and a labeled sentiment corpus to detect the polarity of the opinion words. The above studies have attempted to improve the efficiency of the sentiment analysis either by using a list of predefined product specifications/features or weighting the feature based on its number of occurrences or using a deep learning approach to label features and classify reviews. However, they failed to consider the semantic relationship between these features and the product domain. Furthermore, they did not consider the feature-related synonym, superior, and inferior words as product features to cover the majority of product reviews. Not only that, but they also did not manage the negative words that affect the classification performance.

\section{The Proposed Ontology-based Approach for Feature Level Sentiment Analysis}

The proposed approach seeks to increase the effectiveness of the sentiment analysis process. It employs semantic similarity and Wordnet Ontology in the following. Extracting the product domain features to avoid spam. Extracting the features-related synonyms, superior, and inferior words and using them as product features to consider more reviews rather than those that contain only explicit product features. Not only that, but the approach is also assigned precise sentiment scores by managing negation and using a corpus of labeled sentiments together with the SentiWordnet dictionary.

The proposed approach consists of two major modules: Feature Gathering and Polarity Measurement and operates as follow. Firstly, the frequent noun and noun phrase words are extracted. Secondly, the extracted words are filtered to get the product domain features by using the semantic similarity. After that, for each product feature, the related synonym, superior, and inferior words are obtained based on Wordnet ontology and semantic similarity. Then, the features dictionary is built to differentiate between spam and non-spam reviews. Whereas, the reviews that have a feature or feature synonym/ superior/inferior words go to processing. Next, the opinion words that express the features in reviews are extracted and classified using SentiWordnet and a labeled sentiment corpus. Lastly, the feature-based review summary is produced. The steps of the ontology-based approach for feature level sentiment analysis are shown in

Figure 1. 


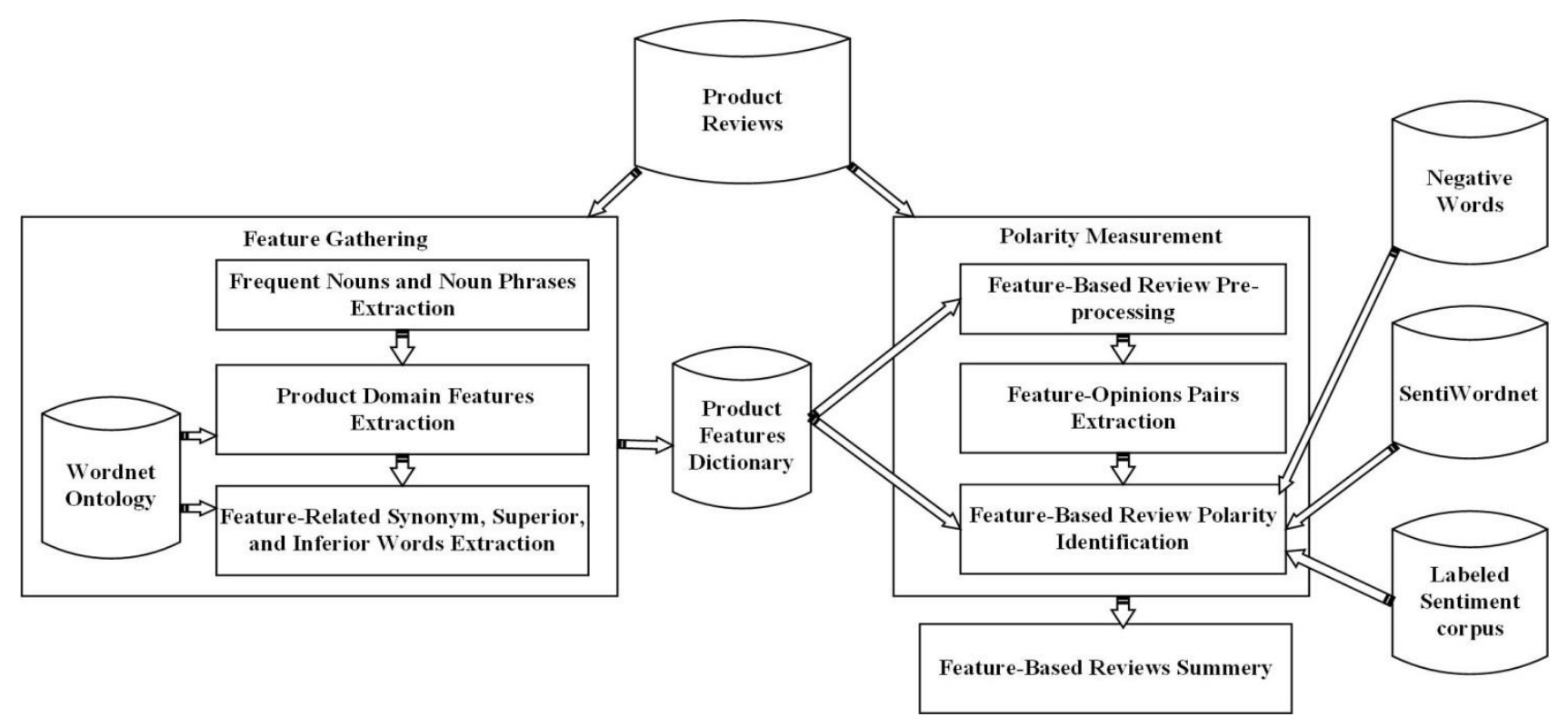

Figure 1: Flowchart of the proposed ontology-based approach for feature level sentiment analysis.

\subsection{Feature gathering}

Wordnet ontology and WU-Palmer semantic similarity measure are involved in this module to build the features dictionary which filters the product reviews before classifying them. The features dictionary consists of the product domain features and their related synonyms, superior, and inferior words. Feature gathering module consists of the following steps:

- Frequent nouns and noun phrases extraction.

- Product domain features extraction.

- Feature-related synonym, superior and inferior words extraction.

\subsubsection{Frequent Nouns and Noun Phrases Extraction}

The proposed approach assumes words that have a part of speech tag equal noun ' $\mathrm{NN}$ ' and noun phrase 'NP' are product features. Therefore, Stanford part of speech tagger is used in this step to tag product reviews. Noun phrase features are considered as a sequence of nouns $\{\langle\mathrm{NN}\rangle\langle\mathrm{NN}\rangle\} /$ $\{\langle\mathrm{NN}\rangle\langle\mathrm{NNS}\rangle\} /\{\langle\mathrm{NNS}\rangle\langle\mathrm{NN}\rangle\} / /\{\langle\mathrm{NNS}\rangle\langle\mathrm{NNS}\rangle\} /$ or an adjective and noun $\{\langle\mathrm{JJ}\rangle\langle\mathrm{NN}\rangle\} /$ $\{\langle\mathrm{JJ}\rangle\langle\mathrm{NNS}\rangle\}$. For example, the following review" great quality picture and video." is tagged into (S great/JJ (NP quality/NN picture/NN) and/CC video/NN. /.). In addition, it has two main features of the Apex DVD player: "quality picture" as a noun phrase feature and "video" as a noun feature. In the frequent nouns and noun phrase extraction step, a predefined threshold is used to obtain the most mentioned noun and noun phrase words in reviews [3].

\subsubsection{Product Domain Features Extraction}

The features that are related to the product domain are obtained in this step using the WU-Palmer semantic similarity exists in Wordnet ontology. The WU-Palmer defines the semantic similarity between the meanings of two words based on their taxonomic depth and their least common 
Subsumer, as shown in Eq. (1). Whereas, the semantic similarity is measured between each candidate product feature retrieved from the previous step and the product domain. For example, the semantic similarity between the word "photo" and the product domain "camera" $=0.6$. Words with a semantic similarity score above the pre-defined threshold are considered as product features [3].

$$
W U P_{\text {Semsim }}=\frac{2 \times \operatorname{depth}(\operatorname{lcs}(c 1, c 2))}{\operatorname{depth}(c 1)+\operatorname{depth}(c 2)}
$$

Where, depth $(\mathrm{c} 1, \mathrm{c} 2)$ is the depth of the product domain meaning and each candidate feature meaning in the taxonomy. Lcs $(\mathrm{c} 1, \mathrm{c} 2)$ is the least common subsume of the meanings $\mathrm{c} 1$ and $\mathrm{c} 2$.

\subsubsection{Feature-Related Synonym, Superior, and inferior Words Extraction}

The feature- related synonym, superior, and inferior words are important in the proposed approach. Because they are regarded as product features in order to increase the recall of reviews. Synonyms of a word are defined as a group of words or phrases that signify exactly or substantially the same word. In addition, superior, and inferior words of a word are the super and sub words or phrases that are linked to the word in Wordnet semantic tree. Therefore, for each product domain feature, its synonyms, superior, and inferior words are retrieved from Wordnet ontology. Then, the WU-Palmer semantic similarity is measured between each product feature and each word exists in the synonyms, superior, and inferior words bag of words. The word with the largest semantic similarity score is considered as the related synonym/ superior/inferior word to the feature. Finally, the features dictionary is built. Algorithm 1 describes how the feature-related synonym, superior, and inferior words are extracted using semantic similarity.

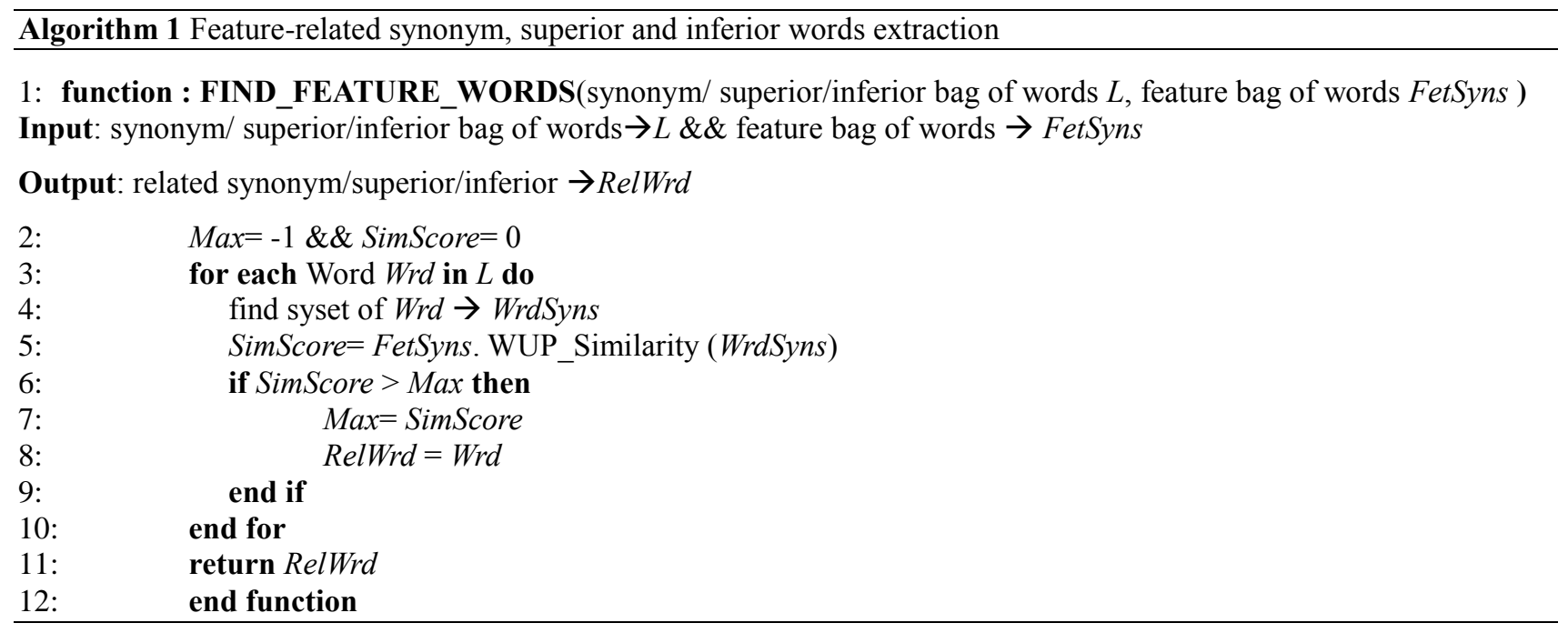

\subsection{Polarity measurement}

The purpose of this module is to produce a feature-based review summary by classifying the product reviews into positive and negative labels based on the product features that exist in the features dictionary. Whereas, the reviews that have a product feature or feature-related synonym/ superior/inferior word are considered in the classification task. The Polarity Measuring Module includes the following steps: 
- Feature-based review pre-processing.

- Feature-opinions pairs extraction.

- Feature-based review polarity identification.

\subsubsection{Feature-Based Review Pre-processing}

The proposed approach considers the review expresses an opinion if it contains a product feature or feature-related synonym/superior/inferior word from the features dictionary. For this type of reviews, the sentence that contains the product feature or feature-related synonym/superior/inferior word is obtained. After that, undesirable words such as stop words, non-alphabetical characters and numbers are deleted from the sentence.

\subsubsection{Feature-Opinions Pairs Extraction}

The proposed approach considers the adverbs, adjectives, verbs, nouns, and all of their forms are opinion words. Therefore, the n-gram technique is used in this step as follow to extract the features opinions pairs. Firstly, the length of the pre-processed sentence "the number of words that exist in a sentence" is verified and then it is split into sized pairs. The pair size depends on the feature length and the sentence length. Therefore, for noun features which have one word: if the sentence length= two, then, the pair size $=$ two. But, if the sentence length exceeds two, then, every pair size $=$ three. However, for noun phrase features which have two or more words, the sentence length must be greater than two words and each pair size $=$ the phrase feature length +1 . Then, the noun feature is looked for in all pairs and once it is found, the part of speech tag and the polarity of its associated words in a pair are verified as they are candidates to be sentiment words. However, if the phrase feature is not found in all pairs, then, it is split into words and each word is considered as a noun feature. Algorithm 2 shows how to extract the opinionated features pairs.

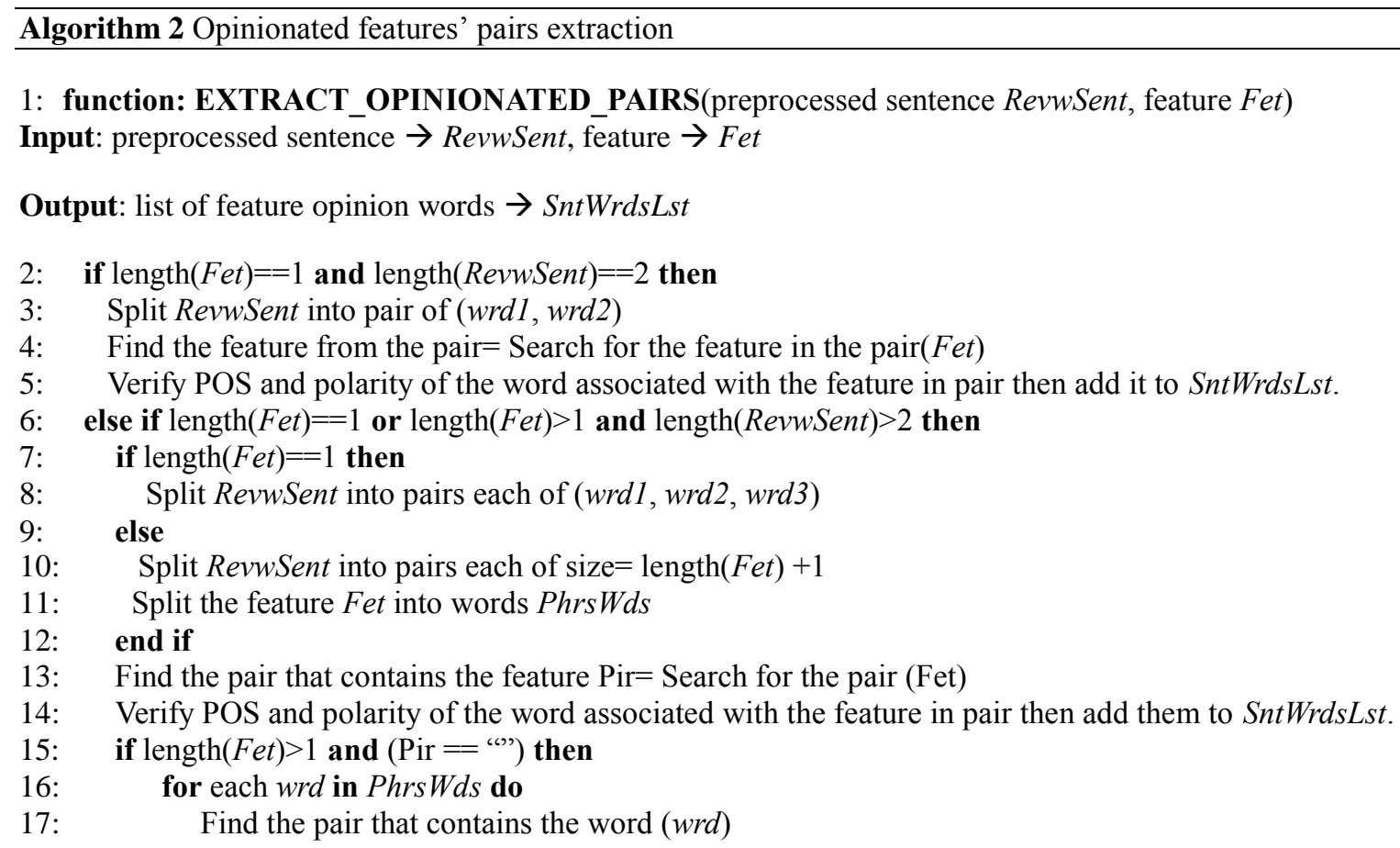


18: $\quad$ Verify POS and and polarity of the words associated with the feature in pair then add them to SntWrdsLst.

19: $\quad$ end for

20: end if

$21:$ end if

22: return SntWrdsLst

23: end function

\subsubsection{Feature-Based Review Polarity Identification}

After extracting the opinionated features pairs, the polarity of each feature-based review is determined and the feature-based review summary is built as shown in

Figure 2. The polarity is measured using the SentiWordnet dictionary and a labeled sentiment corpus which contains a group of well-known labeled opinion words. To get more precise sentiment scores, the proposed approach manages the negative words such as isn't, aren't, no, won't, does not that cause a wrong classification. Whereas negative words change the polarity of opinion words. For example, the next two sentences "I like the sound" and "I don't like the sound" have two different polarity scores. The first sentence is positive, but the word "don't" has inverted the polarity from positive to negative. Algorithm 3 demonstrates how the proposed approach addresses negative words.

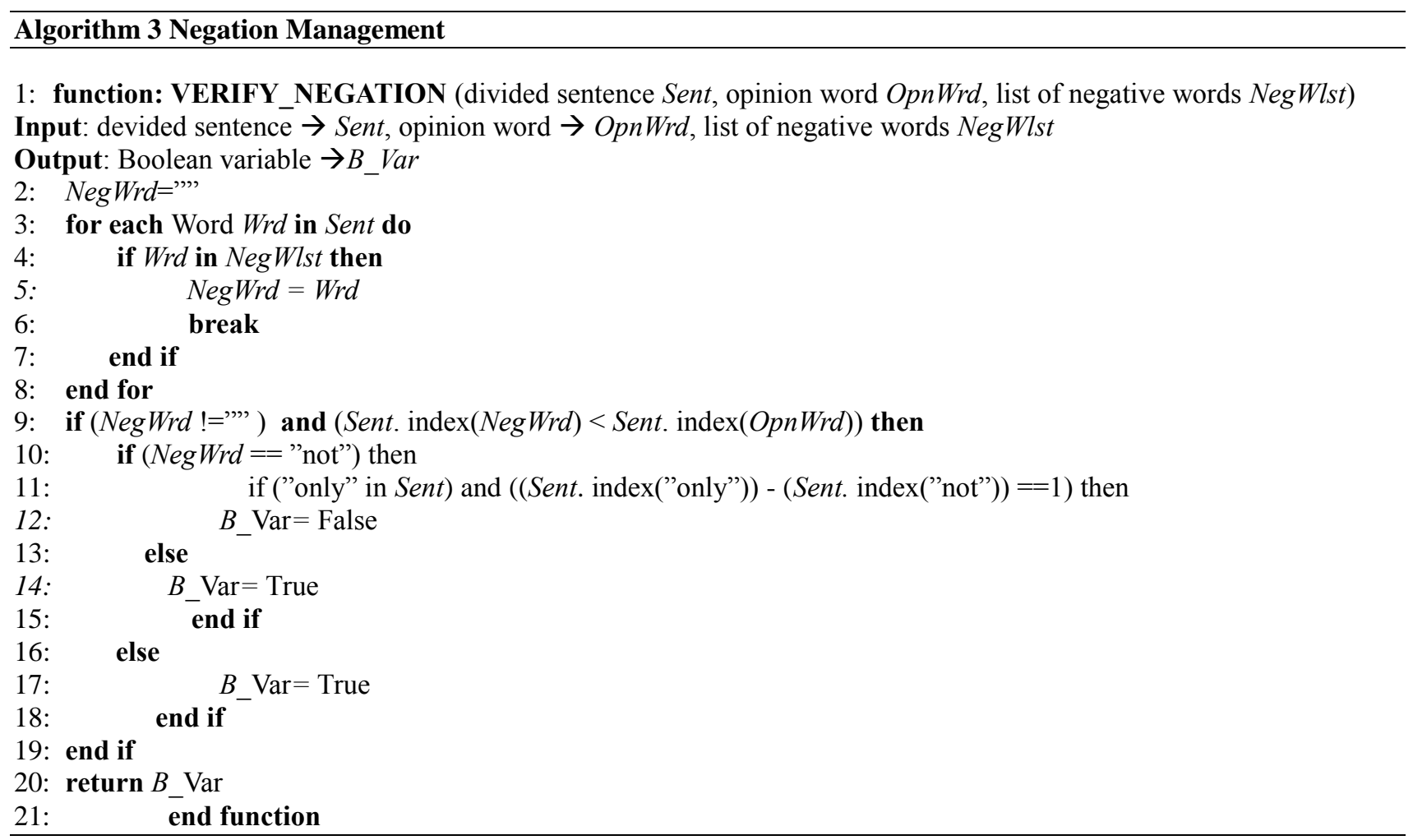




\section{$\underline{\text { Feature-Based Review Summary }}$}

Product Name: Apex AD2600.

$\begin{array}{lcc}\text { Feature } & \text { Pos Reviews } & \text { Neg Reviews } \\ \text { Picture } & 77.8 \% & 22.2 \% \\ \text { Disc } & 46.2 \% & 53.8 \% \\ \text { Remote control } & 50 \% & 50 \% \\ \text { Video output } & 62.5 \% & 37.5 \%\end{array}$

Figure 2: Feature-based review summary.

\section{Evaluation and Results}

This section discusses datasets, performance measures and experimental results used in the present study.

\subsection{Dataset description}

Two benchmark amazon products datasets called Apex AD2600 Progressive-scan DVD player (Apex AD2600) and Nikon Coolpix 4300 (Nikon 4300) are used to evaluate the proposed approach. The datasets are prepared and developed by [17]. The reviews are written as follow in the datasets. Each review is classified into positive and negative by its features. Additionally, the review is assessed from 3(highest) to 1(lowest) and begins after the \#\# sign. Statistics for these datasets are presented in Table 1.

Table 1 Datasets Statistics

\begin{tabular}{|l|l|l|l|l|}
\hline Product dataset & \multirow{2}{*}{ Domain } & \multicolumn{2}{|l|}{ \#Classified Reviews } & \multirow{2}{*}{ \#Features } \\
\cline { 3 - 4 } & & \#Pos & \#Neg & \\
\hline Apex AD2600 & DVD Player & 195 & 236 & 431 \\
\hline Nikon 4300 & Camera & 172 & 31 & 203 \\
\hline
\end{tabular}

\subsection{Performance measures}

Accuracy, precision, recall, and f-measure are used to assess the performance of the proposed approach and defined as follow:

- Accuracy: is the ratio of correctly expected observation to the total observations. 


$$
\text { Accuracy }=\frac{T P+T N}{T P+T N+F P+F N}
$$

- Precision: is the ratio of correctly labeled features to the total of positive labeled features.

$$
\text { Precision }=\frac{T P}{T P+F P}
$$

- Recall (R): is the ratio of correctly labeled positive features to the total labeled features.

$$
\text { Recall }=\frac{T P}{T P+F N}
$$

- F-measure: is a combination of precision and recall.

$$
F-\text { measure }=2 \times \frac{\text { Recall } \times \text { precision }}{\text { Recall }+ \text { Precision }}
$$

In the aforementioned formulas, TP (True-Positive) indicates how many words are correctly labeled as product features. FP (False-Positive) indicates how many words are wrongly labeled as product features. FN (False-Negative) indicates how many words are wrongly labeled as non-product features. TN (True-Negative) indicates how many words are correctly labeled as non-product features.

\subsection{Results and analysis}

The SentiWordnet dictionary and a labeled sentiment corpus are used to evaluate the proposed ontology-based approach for feature sentiment analysis in terms of accuracy, precision, recall, and fmeasure. The evaluation is based on two product datasets which namely Apex AD2600 and Nikon 4300. The results are shown in Table 2 and

Figure 3.

Table 2 The performance of the proposed approach using two amazon products.

\begin{tabular}{|l|l|l|l|l|}
\hline Product Dataset & Accuracy (\%) & Precision (\%) & Recall (\%) & F-measure (\%) \\
\hline Apex AD2600 & 94.5 & 98.6 & 93 & 95.4 \\
\hline Nikon 4300 & 90.3 & 95.8 & 92.6 & 93.4 \\
\hline Average & $92.4 \%$ & $97.2 \%$ & $92.8 \%$ & $94.4 \%$ \\
\hline
\end{tabular}




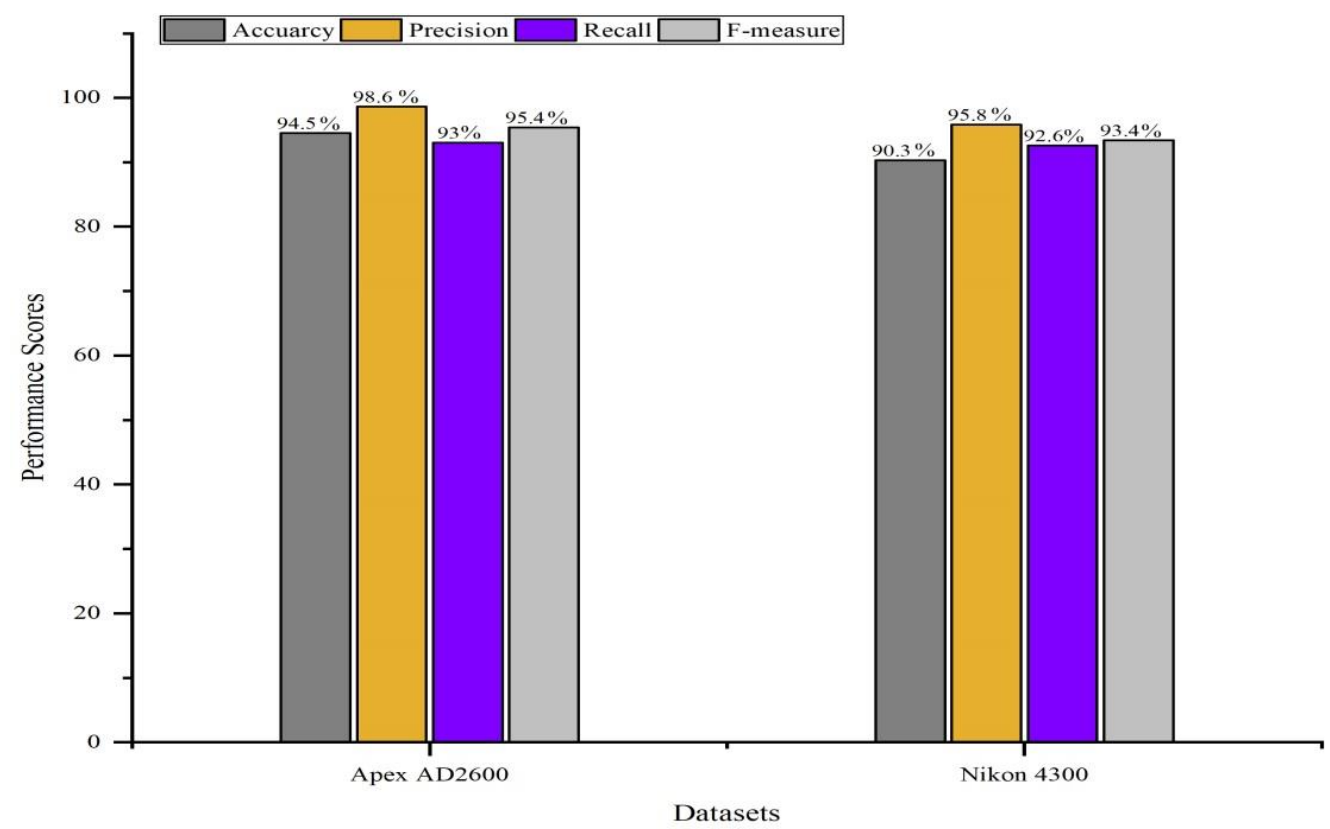

Figure 3: Performance of the proposed approach using two amazon products.

\section{Conclusion and future work}

In this study, an ontology-based approach for feature level sentiment analysis is proposed. The proposed approach used the WU-Palmer semantic similarity measure to obtain the product domain features. Additionally, it considered a feature as a noun or noun phrase. Not only that, but it also labeled the reviews according to different types of features: product domain features, features' synonyms, superior, and inferior words. Moreover, the proposed approach managed the negative words in reviews classification task. SentiWordnet dictionary is used with a labeled sentiment corpus to assign more precise polarity scores. The experimental results showed that the proposed approach achieved promising results in terms of $92.4 \%$ accuracy, $97.2 \%$ precision, $92.8 \%$ recall, and $94.4 \% \mathrm{f}-$ measure. There are a number of enhancements to this study will be discussed going forward. The first enhancement is to combine the synonyms "battery" and "battery life" into "battery". The second one is to address the implicit features that are extracted depending on the review meaning. Finally, taking into account the opinion words strength and suggesting a new approach for allocating more accurate sentiment scores are further improvements.

\section{References}

1. R. Yang, Machine Learning and Deep Learning for Sentiment Analysis over Students' Reviews: An Overview Study, Preprints 2021, 2021020108, 2021, p.1-9.

2. Sonia, Opinion Mining Techniques and Its Applications: A Review, In: Proceedings of First International Conference on Computing, Communications, and Cyber-Security , 2020, pp.549559.

3. E. Aboelela, W. Gad, and R.Ismail, Feature Extraction Using Semantic Similarity, In: Proceedings of the Systems and Informatics on Advanced Intelligent International Conference, 2019, pp.82-91. 
4. A. Ligthart, C. Catal, B. Tekinerdogan, Systematic reviews in sentiment analysis: a tertiary study. Artif Intell Rev (2021). https://doi.org/10.1007/s10462-021-09973-3.

5. A. F. Anees, A. Shaikh, S. Shaikh, Survey paper on sentiment analysis: techniques and challenges, EasyChair Preprint no. 2389, 2020.

6. A. Asmi, T. Ishaya, Negation identification and calculation in sentiment analysis, In: Proceedings of the Second International Conference on Advances in Information Mining and Management, 2012, pp.1-7.

7. D. Gräbner, M. Zanker, G. Fliedl, M. Fuchs, Classification of customer reviews based on sentiment analysis, In: the 19th Conference on Information and Communication Technologies in Tourism (ENTER), 2012, pp.460-470.

8. V. K. Singh, R. Piryani, A. Uddin, P. Waila, Marisha, Sentiment analysis of textual reviews; evaluating machine learning, unsupervised and SentiWordNet approaches, In: the 5th International Conference on Knowledge and Smart Technology (KST), 2013, pp.122-127.

9. S. S. Sohail, J. Siddiqui and R. Ali, Book recommendation system using opinion mining technique, In: Proceedings of 2013 International Conference on Advances in Computing, Communications and Informatics (ICACCI), 2013, pp.1609-1614.

10. P. Liu, S. Joty, H. Meng, Fine-grained opinion mining with recurrent neural networks and word embedding, In: Proceedings of the 2015 conference on empirical methods in natural language processing , 2015, pp.1433-1443.

11. S. Poria, E. Cambria, A. Gelbukh, Aspect extraction for opinion mining with a deep convolutional neural network, Knowledge-Based Systems. 108 (2016) pp.42-49

12. S. S. Sohail, J. Siddiqui and R. Ali, Feature extraction and analysis of online reviews for the recommendation of books using opinion mining technique, Perspectives in Science. 8 (2016) pp.754-756.

13. K. Yang, Y. Cai, D. Huang, J. Li, Z. Zhou and X. Lei, An effective hybrid model for opinion mining and sentiment analysis, In: 2017 IEEE International Conference on Big Data and Smart Computing (BigComp), 2017, pp.465-466.

14. S. S. Sohail, J. Siddiqui and R. Ali, Feature-based opinion mining approach (FOMA) for improved book recommendation, Arabian Journal for Science and Engineering. 43(12) (2018) 8029-8048.

15. H. Xu, B. Liu, L. Shu, P.S. Yu, Double embeddings and CNN-based sequence labeling for aspect extraction, In: Proceedings of the 56th annual meeting of the association for computational linguistics, 2018, pp. 592-601

16. A. Da'u, N. Salim, I. Rabiu, A. Osman, Weighted aspect-based opinion mining using deep learning for recommender system, Expert Systems with Applications. 140 (2020) pp.1-12.

17. M. Hu, B. Liu, Mining and summarizing customer reviews, In: Proceedings of the tenth ACM SIGKDD international conference on Knowledge discovery and data mining, 2004, pp.168-177. 